“ (C) 2017 IEEE. Personal use of this material is permitted. Permission from IEEE must be obtained for all other uses, in any current or future media, including

reprinting/republishing this material for advertising or promotional purposes, creating new collective works, for resale or redistribution to servers or lists, or reuse of any copyrighted component of this work in other works." 


\section{Message from the IEEE TrustCom 2017 Program Chairs}

Welcome to the 16th IEEE International Conference on Trust, Security and Privacy in Computing and Communications (TrustCom 2017), Sydney, Australia, August 1-4, 2017. On behalf of the Organizing Committee of TrustCom 2017, we would like to express our sincere thanks and warm welcome to all our delegates and guests in Sydney.

With the rapid development and increasing complexity of computer systems and communication networks, user requirements for trust, security and privacy are becoming more and more demanding. Therefore, there is a grand challenge that traditional security technologies and measures may not meet user requirements in open, dynamic, heterogeneous, mobile, wireless, and distributed computing environments. Thus, we need to build systems and networks in which various applications allow users to enjoy more comprehensive services while preserving trust, security and privacy at the same time. As useful and innovative technologies, trusted computing and communications are attracting researchers with more and more attention.

TrustCom 2017 follows the success of TrustCom-16 in Tianjin, China, TrustCom-15 in Helsinki, Finland, TrustCom-14 in Beijing, China and so on. The conference aims at bringing together researchers and practitioners throughout the world working on trusted computing and communications, with regard to trust, security, privacy, reliability, dependability, survivability, availability, and fault tolerance aspects of computer systems and networks, and providing a forum to present and discuss emerging ideas and trends in this highly challenging research field. TrustCom 2017 consists of the main conference, 5 workshops/symposiums, and a total of 127 high-quality peer reviewed papers. The final acceptance decisions on the submitted papers were made after a rigorous review process conducted by the Technical Program Committee (TPC).

We would like to express our sincere gratitude to the Program Co-Chairs, Track Co-Chairs and Program Committee members for their effective work and great effort. We also greatly appreciate the cooperation, guidance, and help from the General Chairs, the Steering Committee, the Publicity Chairs, and the Finance Chair. The support from the IEEE Computer Society's Technical Committee on Scalable Computing (TCSC) is also greatly appreciated.

We would like to thank you all for participating in TrustCom 2017, and hope you to find the conference stimulating and interesting for your research and professional activities.

Priyadarsi Nanda - University of Technology Sydney, Australia

Yang Xiang - Deakin University, Melbourne, Australia

Yi Mu - University of Wollongong, Australia

Program Chairs of TrustCom 2017 